\title{
On the equilibrium rotation of Hot Jupiters in eccentric and excited orbits
}

\author{
Alexandre C. M. Correia ${ }^{1,2}$ \\ ${ }^{1}$ Department of Physics, I3N, University of Aveiro, \\ Campus Universitário de Santiago, 3810-193 Aveiro, Portugal \\ email: correia@ua.pt \\ ${ }^{2}$ Astronomie et Systèmes Dynamiques, IMCCE-CNRS UMR 8028, \\ 77 Avenue Denfert-Rochereau, 75014 Paris, France
}

\begin{abstract}
Hot-Jupiters are a common sub-class of exoplanets, which are enough close to the star to undergo tidal dissipation. The continuous action of tides modify the rotation of the planets until an equilibrium situation is reached. It is often assumed that synchronous motion is the most probable outcome of tidal evolution, since synchronous rotation is observed for the majority of the satellites in the Solar System. This is true for circular orbits, but when the orbits are eccentric, tidal effects are stronger when the planets are closer to the star, and therefore, the rotation rate tends to equalize the orbital speed rate at the pericenter (which is faster than synchronous rotation). An additional complication arises if the eccentricity is not constant and undergoes periodic perturbations from an external companion. Here we obtain an expression for the equilibrium rotation of Hot-Jupiters undergoing tidal dissipation and planetary perturbations. We show that for these planets, the equilibrium rotation rate is faster than for non-perturbed eccentric orbits.
\end{abstract}

Keywords. planetary systems, celestial mechanics, methods: analytical

\section{Introduction}

At present, about half of the exoplanets that have been detected are close-in planets with semi-major axis smaller than 0.4 AU (http://exoplanet.eu/). This percentage is biased, since close-in planets are easily detected by radial velocity and transit methods (the two methods with greater success in discovering exoplanets). However, it shows that massive planets are often found within a distance to the star equivalent to the Sun-Mercury distance. As a consequence, these planets undergo significant tidal interactions with the star, resulting that their spins will be slowly modified.

The ultimate stage for tidal evolution corresponds to a low obliquity and synchronous rotation, a configuration where the rotation rate coincides with the orbital mean motion, since the synchronous equilibrium corresponds to the minimum of dissipation of energy. However, when the eccentricity is different from zero some other configurations are possible, such as the $3 / 2$ spin-orbit resonance observed for the planet Mercury (e.g., Correia \& Laskar 2004) or the chaotic rotation of Hyperion (Wisdom et al. 1984). Indeed, for eccentric orbits the rotation rate of the planet tends to equalize the orbital speed rate at the pericenter, which is faster than synchronous rotation. About $1 / 3$ of the planets with a semi-major axis smaller than 0.4 have an eccentricity above 0.1 , so it is important to understand how their equilibrium rotation rates will be modified.

Additional effects may also contribute to the final evolution of the spin, such as atmospheric tides or planetary perturbations. The effect of a dense atmosphere has been studied in Correia et al. (2008). Here we will focus on the effect of planetary perturbations on the eccentricity of the close-in planet. A similar problem has been studied for 
the spin of Mercury (Correia \& Laskar 2004), but in that case we also needed to take into account the contribution of the asymmetric equatorial moments of inertia $C_{22}$, which is responsible for capture in spin-orbit resonances. Here we will look at the behavior of Jupiter and Neptune-like planets for which the second order harmonics of the gravity field are close to zero (e.g. Jacobson 2001), leading to insignificant chances of capture. We thus expect that the final rotation of these planets is controlled uniquely by the equilibrium tide.

\section{Equations of motion}

We will adopt here a viscous tidal model, with a linear dependence on the tidal frequency, which is suitable to study slow rotations near the equilibrium, and is also valid for any value of the eccentricity (e.g. Mignard 1979). Tidal dissipation and core-mantle friction drive the planet's obliquity (the angle between the equator and the orbital plane) close to zero (e.g. Correia et al. 2003), so we will assume zero degree obliquity throughout this study for simplicity. The tidal contribution to the rotation rate, $\omega$, is then given by (e.g. Correia \& Laskar 2010):

$$
\dot{\omega}=-K[\Omega(e) \omega-N(e) n]
$$

with

$$
\begin{gathered}
\Omega(e)=\frac{1+3 e^{2}+3 e^{4} / 8}{\left(1-e^{2}\right)^{9 / 2}} \\
N(e)=\frac{1+15 e^{2} / 2+45 e^{4} / 8+5 e^{6} / 16}{\left(1-e^{2}\right)^{6}}
\end{gathered}
$$

and

$$
K=3 n \frac{k_{2}}{\xi Q}\left(\frac{R}{a}\right)^{3}\left(\frac{m_{0}}{m}\right),
$$

where $\xi$ is a structure constant, $k_{2}$ is the second Love number, $Q$ is the dissipation quality factor, and $n, a, R, m$, and $m_{0}$ are the mean motion, the semi-major axis, the radius of the planet, its mass, and the mass of the star, respectively.

For a non-constant varying eccentricity $e(t)$, the solution of equation $(2.1)$ is given by (Correia \& Laskar 2004):

$$
\omega(t)=\left(\omega(0)+n K \int_{0}^{t} N(e(\tau)) g(\tau) d \tau\right) / g(t),
$$

with

$$
g(t)=\exp \left(K \int_{0}^{t} \Omega(e(\tau)) d \tau\right)
$$

\section{Approximations}

The eccentricity of a planet that is disturbed by the gravitational perturbations of additional planetary companions can be expressed as a sum of quasi-periodic terms (e.g. Laskar 1988):

$$
e(t)=\sum_{i} e_{i} \mathrm{e}^{\mathrm{i}\left(\nu_{i} t+\phi_{i}\right)}
$$

where $e_{i}$ is an amplitude, $\nu_{i}$ is the frequency of the perturbation, and $\phi_{i}$ is a phase angle. When there is a single companion, its orbit lies in the same plane as the planet (i.e., the 
system is coplanar), and we take into account only the linear terms of the perturbation, the eccentricity variations can be simply written as:

$$
e(t)=e_{0}+\Delta e \cos (\nu t+\phi) .
$$

The expressions of $\Omega(e)$ (Eq. 2.2) and $N(e)$ (Eq. 2.3) are functions of $e^{2}$. Since $0 \leqslant e \leqslant$ 1 , we can develop these two functions in power series of $e^{2}$ as

$$
\begin{aligned}
& \Omega(e)=\sum_{j=0}^{+\infty} \Omega_{j} e^{2 j}=1+\frac{15}{2} e^{2}+\frac{105}{4} e^{4}+\ldots, \\
& N(e)=\sum_{j=0}^{+\infty} N_{j} e^{2 j}=1+\frac{27}{2} e^{2}+\frac{573}{8} e^{4}+\ldots
\end{aligned}
$$

Because $e(t)$ can be expressed as a sum of quasi-periodic terms (Eq.3.1), so does the functions $\Omega(e)$ and $N(e)$, as:

$$
\Omega(e)=\sum_{i, j} \Omega_{i j} e^{\mathrm{i} j\left(\nu_{i} t+\phi_{i}\right)}, \quad N(e)=\sum_{i, j} N_{i j} e^{\mathrm{i} j\left(\nu_{i} t+\phi_{i}\right)} .
$$

Considering the case of a single companion in a coplanar orbit (Eq. 3.2), these expressions can be obtained directly from equations (3.3) and (3.4), with

$$
e^{2 j}=e_{0}^{2 j} \sum_{k=0}^{2 j} \sum_{l=0}^{k}\left(\begin{array}{c}
2 j \\
k
\end{array}\right)\left(\begin{array}{c}
k \\
l
\end{array}\right)\left(\frac{\Delta e}{2 e_{0}}\right)^{k} \mathrm{e}^{\mathrm{i}(k-2 l)(\nu t+\phi)} .
$$

Henceforward we will restrict our analysis to the case of a single companion in a coplanar orbit, but our conclusions can be easily extended to the general situation given by equations (3.1) and (3.5).

\section{Averaging}

Periodic varying quantities can be averaged in order to obtain their mean values. This can be particularly interesting when the eccentricity variations occur in a time-scale that is shorter than the time-scale for tidal variations $(\nu \gg K)$, which is often the case. Thus,

$$
\overline{e(t)}=\frac{2 \pi}{\nu} \int_{0}^{\frac{2 \pi}{\nu}} e(t) d t=e_{0}
$$

and

$$
\begin{aligned}
\overline{e^{2 j}} & =e_{0}^{2 j} \sum_{k=0}^{2 j} \sum_{l=0}^{k}\left(\begin{array}{c}
2 j \\
k
\end{array}\right)\left(\begin{array}{c}
k \\
l
\end{array}\right)\left(\frac{\Delta e}{2 e_{0}}\right)^{k} \frac{2 \pi}{\nu} \int_{0}^{\frac{2 \pi}{\nu}} \mathrm{e}^{\mathrm{i}(k-2 l)(\nu t+\phi)} d t \\
& =e_{0}^{2 j} \sum_{k=0}^{2 j} \sum_{l=0}^{k}\left(\begin{array}{c}
2 j \\
k
\end{array}\right)\left(\begin{array}{c}
k \\
l
\end{array}\right)\left(\frac{\Delta e}{2 e_{0}}\right)^{k} \delta_{k, 2 l} \mathrm{e}^{\mathrm{i}(k-2 l) \phi} \\
& =e_{0}^{2 j} \sum_{l=0}^{j}\left(\begin{array}{c}
2 j \\
2 l
\end{array}\right)\left(\begin{array}{c}
2 l \\
l
\end{array}\right)\left(\frac{\Delta e}{2 e_{0}}\right)^{2 l} .
\end{aligned}
$$

Finally, using the above quantity in the expressions of $\Omega(e)$ and $N(e)$ (Eq. 3.3 and 3.4), we get: 


$$
\begin{aligned}
\bar{\Omega}=\overline{\Omega(e)} & =\sum_{j=0}^{+\infty} \sum_{l=0}^{j} \frac{\Omega_{j} e_{0}^{2 j}(2 j) !}{(2 j-2 l) !(l !)^{2}}\left(\frac{\Delta e}{2 e_{0}}\right)^{2 l} \\
& =1+\frac{15}{2} e_{0}^{2}+\frac{105}{4} e_{0}^{4}+\frac{15}{4} \Delta e^{2}+\frac{315}{4} e_{0}^{2} \Delta e^{2}+\frac{315}{32} \Delta e^{4}+\ldots \\
\bar{N}=\overline{N(e)} & =\sum_{j=0}^{+\infty} \sum_{l=0}^{j} \frac{N_{j} e_{0}^{2 j}(2 j) !}{(2 j-2 l) !(l !)^{2}}\left(\frac{\Delta e}{2 e_{0}}\right)^{2 l} \\
& =1+\frac{27}{2} e_{0}^{2}+\frac{573}{8} e_{0}^{4}+\frac{27}{4} \Delta e^{2}+\frac{1719}{8} e_{0}^{2} \Delta e^{2}+\frac{1719}{64} \Delta e^{4}+\ldots
\end{aligned}
$$

\section{Implications}

For a constant eccentricity $e(t)=e_{0}$, the solution of equation (2.1) becomes

$$
\omega(t)=\omega_{e_{0}}+\omega(0) \mathrm{e}^{-K \Omega\left(e_{0}\right) t},
$$

the equilibrium being achieved when $t \gg 1 /\left(K \Omega\left(e_{0}\right)\right)$ for $\omega(t)=\omega_{e_{0}}=C t e$, where

$$
\frac{\omega_{e_{0}}}{n}=\frac{N\left(e_{0}\right)}{\Omega\left(e_{0}\right)}=1+6 e_{0}^{2}+\frac{3}{8} e_{0}^{4}+\ldots
$$

This equilibrium is often presented as the equilibrium rotation rate for planets in eccentric orbits, but in fact, this is only true for unperturbed orbits. Since unperturbed eccentricities of close-in planets are quickly damped to zero, contrarily to eccentricities of close-in planets with companions (Mardling 2007), most of the observed close-in planets with some eccentricities probably have companions, and therefore forced eccentricities.

For a non constant eccentricity $e(t)$, the solution of equation (2.1) is given by expression (2.5), which cannot be solved without some approximations. Assuming that $e(t)$ is simply given by expression (3.2), we have for $t \gg 1 /(K \bar{\Omega})$ and $\nu / K \gg 1$ :

$$
g(t)=\exp \left(K \int_{0}^{t} \Omega(e(\tau)) d \tau\right) \approx \exp (K \bar{\Omega} t)
$$

and

$$
\frac{\omega(t)}{n} \approx K \mathrm{e}^{-K \bar{\Omega} t} \int_{0}^{t} N(e(\tau)) \mathrm{e}^{K \bar{\Omega} \tau} d \tau
$$

Thus, replacing equations (3.4) and (3.6) in the above expression, gives:

$$
\frac{\omega(t)}{n}=\sum_{j=0}^{+\infty} N_{j} e_{0}^{2 j} \sum_{k=0}^{2 j} \sum_{l=0}^{k}\left(\begin{array}{c}
2 j \\
k
\end{array}\right)\left(\begin{array}{c}
k \\
l
\end{array}\right)\left(\frac{\Delta e}{2 e_{0}}\right)^{k} \Phi_{k l}(t),
$$

where

$$
\begin{aligned}
\Phi_{k l}(t) & =K \mathrm{e}^{-K \bar{\Omega} t} \int_{0}^{t} \mathrm{e}^{\mathrm{i}(k-2 l)(\nu \tau+\phi)+K \bar{\Omega} \tau} d \tau \\
& =\frac{K \mathrm{e}^{\mathrm{i}(k-2 l)(\nu t+\phi)}}{\mathrm{i}(k-2 l) \nu+K \bar{\Omega}}=\frac{\cos \left[(k-2 l)(\nu t+\phi)-\psi_{k l}\right]}{\sqrt{(k-2 l)^{2} \nu^{2} / K^{2}+\bar{\Omega}^{2}}},
\end{aligned}
$$

with

$$
\tan \psi_{k l}=\frac{(k-2 l) \nu}{K \bar{\Omega}}
$$




\section{1. average rotation rate}

Both $\omega(t)$ and $\Phi_{k l}(t)$ are periodic functions, with period $2 \pi / \nu$. One may then compute their average value,

$$
\overline{\Phi_{k l}(t)}=\frac{2 \pi}{\nu} \int_{0}^{\frac{2 \pi}{\nu}} \frac{K \mathrm{e}^{\mathrm{i}(k-2 l)(\nu t+\phi)}}{\mathrm{i}(k-2 l) \nu+K \bar{\Omega}} d t=\frac{\delta_{k, 2 l}}{\bar{\Omega}},
$$

and

$$
\begin{aligned}
\frac{\overline{\omega(t)}}{n} & =\sum_{j=0}^{+\infty} N_{j} e_{0}^{2 j} \sum_{k=0}^{2 j} \sum_{l=0}^{k}\left(\begin{array}{c}
2 j \\
k
\end{array}\right)\left(\begin{array}{c}
k \\
l
\end{array}\right)\left(\frac{\Delta e}{2 e_{0}}\right)^{k} \frac{\delta_{k, 2 l}}{\bar{\Omega}} \\
& =\frac{1}{\bar{\Omega}} \sum_{j=0}^{+\infty} N_{j} e_{0}^{2 j} \sum_{l=0}^{j}\left(\begin{array}{c}
2 j \\
2 l
\end{array}\right)\left(\begin{array}{c}
2 l \\
l
\end{array}\right)\left(\frac{\Delta e}{2 e_{0}}\right)^{2 l}=\frac{\bar{N}}{\bar{\Omega}} \\
& =\frac{N\left(e_{0}\right)}{\Omega\left(e_{0}\right)}+3 \Delta e^{2}+\frac{729}{8} e_{0}^{2} \Delta e^{2}+\frac{369}{64} \Delta e^{4}+\ldots,
\end{aligned}
$$

that is, the average value of the rotation rate $\bar{\omega}=\overline{\omega(t)} \geqslant \omega_{e_{0}}$ is always faster than the value of the equilibrium rotation rate for the average value of the eccentricity, $e_{0}$ (Eq. 5.2). The difference depends on the amplitude of the eccentricity librations $\Delta e$. We then conclude that for orbits undergoing eccentricity excitations, the equilibrium rotation rate of the planet is faster than for non-perturbed eccentric orbits.

\section{2. weak tidal dissipation $(\nu \gg K)$}

In general, the time-scale for the eccentricity variations is shorter than the time-scale for the tidal damping $(\nu \gg K)$. In this limit situation, we may neglect the contributions from $K \bar{\Omega}$ in expression (5.6), except those from terms with $k=2 l$, which correspond to the non-periodic terms contributing to the average $\bar{\omega}$ (Eq. 5.9). Thus, retaining only the terms of minimal frequency, $k-2 l=0$ and $k-2 l=1$, we get

$$
\frac{\omega(t)}{n} \approx \frac{\bar{N}}{\bar{\Omega}}+\frac{K}{\nu}\left(\frac{\Delta e}{2 e_{0}}\right) A_{1} \cos (\nu t+\phi-\pi / 2),
$$

with

$$
A_{1}=\sum_{j=0}^{+\infty} N_{j} e_{0}^{2 j} \sum_{l=0}^{j-1}\left(\begin{array}{c}
2 j \\
2 l+1
\end{array}\right)\left(\begin{array}{c}
2 l+1 \\
l
\end{array}\right)\left(\frac{\Delta e}{2 e_{0}}\right)^{2 l} .
$$

We then conclude that the rotation rate of a planet in an eccentric and excited orbit is given by the average value of the rotation rate, $\bar{\omega}$, plus a sinusoidal function similar to the eccentricity (Eq. 3.2), but with smaller amplitude and a phase delay of $\pi / 2$. When we include the remaining harmonics the behavior is identical, only the shape of the periodic signal is modified (Eq. 5.6). As the magnitude of tidal effects increase, the amplitude of the periodic variations becomes larger, and the phase delay becomes smaller than $\pi / 2$.

Table 1. Initial parameters for the HD 11964 system (Wright et al. 2009).

\begin{tabular}{|c|c|c|c|c|c|c|c|c|}
\hline & \multicolumn{8}{|c|}{ HD $11964\left(m_{0}=1.1 M_{\odot}\right)$} \\
\hline $\begin{array}{c}\text { parameter } \\
\text { (unit) }\end{array}$ & $\begin{array}{c}a \\
(\mathrm{AU})\end{array}$ & $e$ & $\begin{array}{c}m \\
\left(M_{\text {Jup }}\right)\end{array}$ & $\begin{array}{c}P_{\text {rot }} \\
\text { (day) }\end{array}$ & $\begin{array}{c}R \\
\left(\times 10^{6} \mathrm{~m}\right)\end{array}$ & $\xi$ & $k_{2}$ & $\begin{array}{c}Q \\
\left(\times 10^{3}\right)\end{array}$ \\
\hline $\begin{array}{l}\text { planet }(b) \\
\text { planet }(c)\end{array}$ & $\begin{array}{c}3.16 \\
0.229\end{array}$ & 0.041 & 0.622 & - & - & - & - & - \\
\hline
\end{tabular}




\section{Applications}

We will now apply the results derived in previous sections to the planet HD $11964 \mathrm{c}$. The planetary system around HD 11964 is composed of two planets with minimum masses $m_{c}=25 M_{\oplus}$ (planet $c$ ) and $m_{b}=0.62 M_{\mathrm{Jup}}$ (planet $b$ ), at $a_{c}=0.229 \mathrm{AU}$ and $a_{b}=$ 3.16 AU, respectively (Wright et al. 2009, Table 1). The orbit of the planet $c$ also presents an eccentricity of about 0.3 and is enough close to the star to undergo substantial tidal

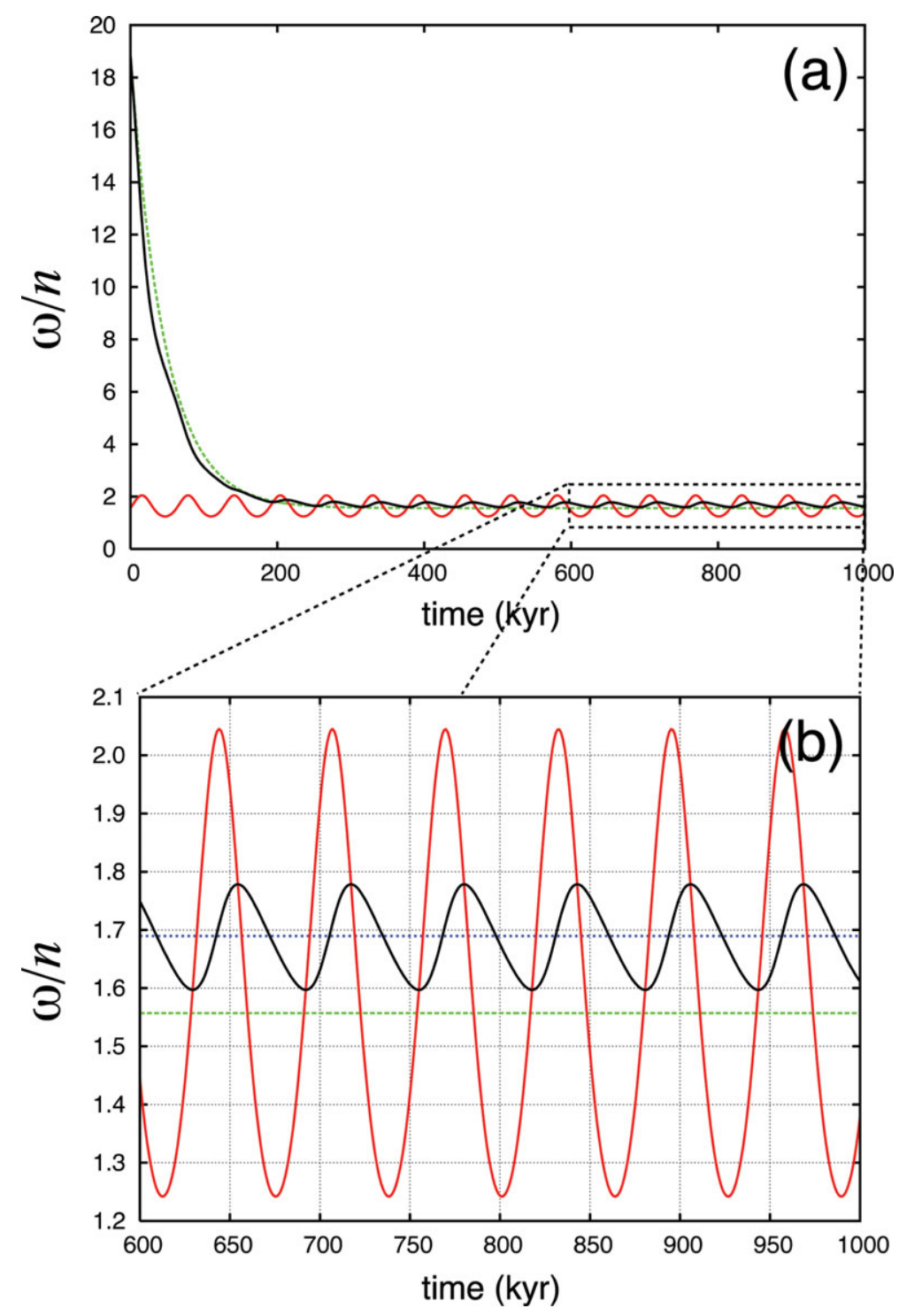

Figure 1. Evolution of the rotation rate with time, starting with the parameters from Table 1, using a model with a varying eccentricity (Eq. 6.1, black solid curve), and a model with constant eccentricity $\left(e_{0}=0.3\right.$, green dashed curve). We also plot the instantaneous value of the ratio $N(e) / \Omega(e)$ for the varying eccentricity model (red solid curve). We observe that in the case of a constant eccentricity, the rotation rate stabilizes in the equilibrium value $\omega_{e_{0}} / n=1.5571$, while in the case of a varying eccentricity, the rotation rate presents a periodic oscillations that follows the equilibrium ratio $N(e) / \Omega(e)$ with a smaller amplitude and a delayed phase angle (Eq.5.10). These oscillations are effectuated around a mean value given by $\bar{\omega} / n=1.6896$ (blue dotted line). 
dissipation. Therefore, this is a good system to illustrate our theoretical results. The average distance between the two planets is relatively high, so if the two orbits are coplanar the eccentricity of the inner body only experiences small oscillations. However, the mutual inclination between the planets is unknown. Veras \& Ford (2010) performed extensive n-body simulations for this system and concluded that it is stable for very high mutual inclinations, which can excite significantly the eccentricity of the inner orbit. We will thus assume that the eccentricity of the planet $c$ can be written in the form of equation (3.2) with $\Delta e=0.1$ and $\nu=10^{-4} \mathrm{yr}^{-1}$ :

$$
e(t)=0.3+0.1 \sin \left(10^{-4} t_{[\mathrm{yr}]}\right) \text {. }
$$
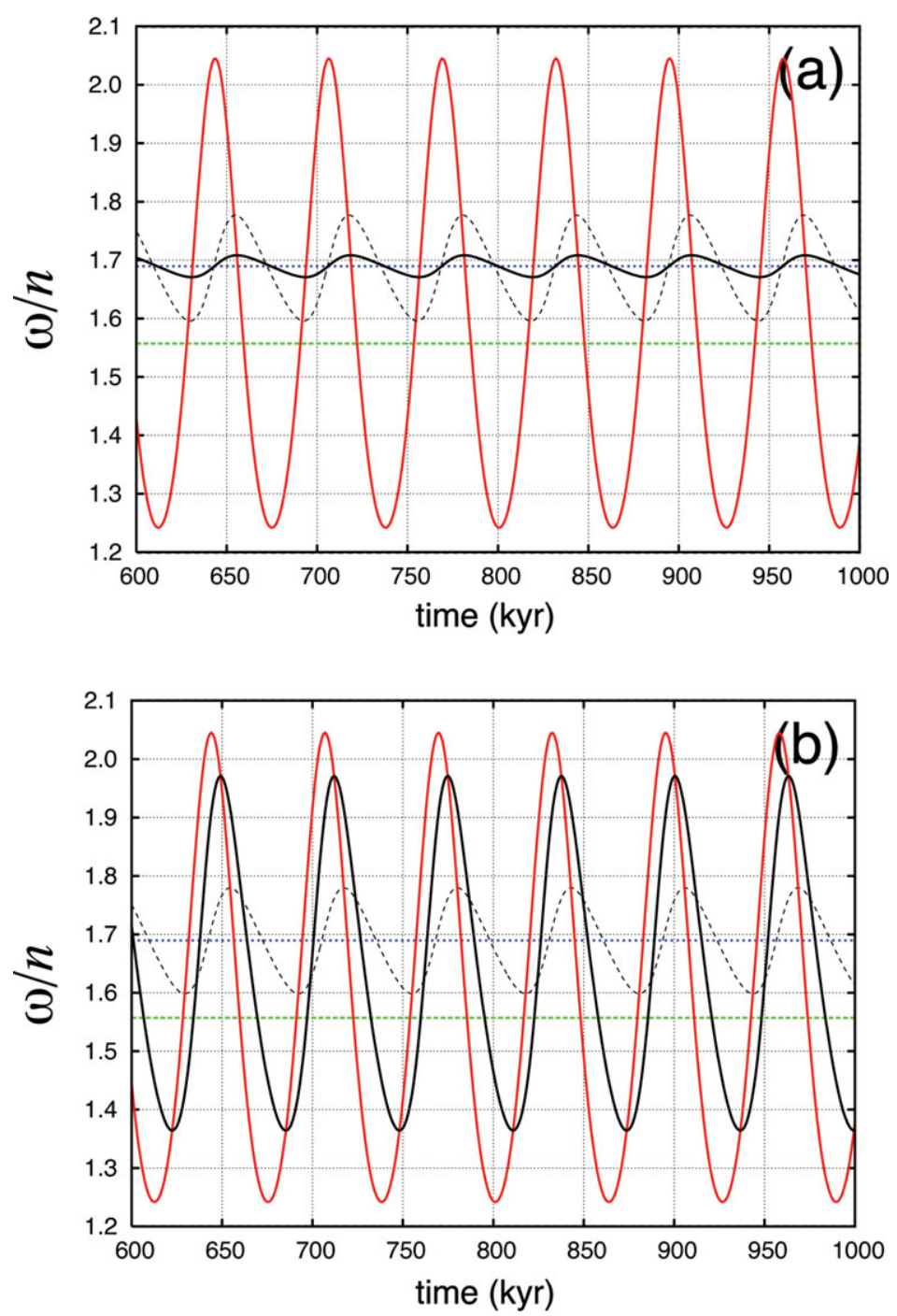

Figure 2. Same as Fig. 1b, but with $Q=2.5 \times 10^{4}$ (a) and $Q=10^{3}$ (b). In the case of a weaker dissipation (a), we observe that the rotation rate shows only very small amplitude librations around the averaged value $\bar{\omega} / n=1.6896$ (blue dotted line). In the case of a stronger dissipation (b), we observe that the rotation rate oscillations closely follow the instantaneous equilibrium rotation given by $N(e) / \Omega(e)$ (red solid line). 
In Figure 1 we plot the evolution of the rotation rate of the planet, starting with a rotation period of 2 days, that is, $\omega(0) / n \approx 18.9$, and using a dissipation factor identical to Jupiter, $Q=5 \times 10^{3}$ (Table 1 ). We plot the evolution of $\omega(t) / n$ using the varying eccentricity given by the expression (6.1) (Eq. 2.1, black curve), but also using a constant eccentricity $e_{0}=0.3$ (Eq. 5.1, green curve). We observe that the evolution of the rotation rate is identical for two situations during the initial damping (Fig. 1a). We also plot the instantaneous value of the ratio $N(e) / \Omega(e)$ for the varying eccentricity (red curve). After about $0.2 \mathrm{Myr}$, the rotation rate is fully damped and enter in an equilibrium regime. In the case of a constant eccentricity, the rotation rate stabilizes in the equilibrium value $\omega_{e_{0}} / n=1.5571$ (Eq. 5.2), while in the case of a varying eccentricity, the rotation rate presents a periodic oscillations that follows the equilibrium ratio $N(e) / \Omega(e)$ with a smaller amplitude and a delayed phase angle (Eq.5.10, Fig. 1b). These oscillations are effectuated around a mean value given by $\bar{\omega} / n=1.6896$ (Eq. 5.9, blue straight line), which is clearly above the rotation rate for constant eccentricity, that is, $\bar{\omega}>\omega_{e_{0}}$.

In Figure 2 we plot the final evolution of the rotation rate (same as Fig. 1b), but for different values of the tidal dissipation factor: (a) $Q=2.5 \times 10^{4}$, i.e., the dissipation is five times weaker; (b) $Q=10^{3}$, i.e., the dissipation is five times more efficient. In the case of a weaker dissipation we observe that the rotation rate shows only very small amplitude librations around the averaged value, that is, $\omega(t) \approx \bar{\omega}$ (Fig. 2a). This behavior is in agreement with expression $(5.10)$, since $K / \nu \approx 0$. In the case of a stronger dissipation we observe that the rotation rate oscillations closely follow the instantaneous equilibrium rotation given by $N(e) / \Omega(e)$. In a limit situation for which $K \gg \nu$, we would have $\omega(t) / n \approx N(e) / \Omega(e)$, since the eccentricity can be considered constant with respect to tidal evolution (Eq. 5.2).

\section{Conclusions}

In these paper we obtained an expression for the equilibrium rotation of Hot-Jupiters undergoing tidal dissipation and planetary perturbations on its orbit. We show that the equilibrium rotation rate is faster than the synchronous rotation, but also than the equilibrium for non-perturbed eccentric orbits (Eq. 5.2). Indeed, the rotation rate presents small oscillations with the same periodicity of the eccentricity around an average value $\bar{\omega}>\omega_{e_{0}}$, where $\omega_{e_{0}}$ is the equilibrium value for constant eccentricity. In particular, for a regular sinusoidal perturbation of the eccentricity with a small amplitude $\Delta e$ (Eq. 3.2), we show that the mean equilibrium rotation rate is given by:

$$
\bar{\omega}=\omega_{e_{0}}+3 \Delta e^{2}+\frac{729}{8} e_{0}^{2} \Delta e^{2}+\frac{369}{64} \Delta e^{4}+\mathcal{O}\left(\Delta e^{6}\right) .
$$

\section{References}

Correia, A. C. M. \& Laskar, J. 2004, Nature, 429, 848

Correia, A. C. M. \& Laskar, J. 2010, Icarus, 205, 338

Correia, A. C. M., Laskar, J., \& Néron de Surgy, O. 2003, Icarus, 163, 1

Correia, A. C. M., Levrard, B., \& Laskar, J. 2008, A\&A 488, L63

Jacobson, R. A. 2001, BAAS, 33, 1039

Laskar, J. 1988, A\&A, 198, 341

Mignard, F. 1979, Moon and Planets, 20, 301

Veras, D. \& Ford, E. B. 2010, ApJ, 715, 803

Wisdom, J., Peale, S. J., \& Mignard, F. 1984, Icarus, 58, 137

Wright, J. T., Upadhyay., S. Marcy, G. W., et al. 2009, ApJ, 693, 1084 